$\xi=$ 国

\title{
Generalized Statistical Modeling Maternal Infant HIV Transmission with Constant Hazard Rate
}

\author{
S.S. Shende ${ }^{1 *}$, M.M. Kale ${ }^{2}$, N. Gupte ${ }^{3}$ \\ ${ }^{l}$ Department of Statistics, Fergusson College, Pune, India \\ ${ }^{2}$ Department of Statistics, Savitribai Phule Pune University, Pune, India \\ ${ }^{3}$ Johns Hopkins University, Division of Infectious Diseases, School of Medicine, Baltimore, USA \\ *Corresponding author E-mail: subhashshende@gmail.com
}

\begin{abstract}
Knowledge of the timing of perinatal transmission of Human Immunodeficiency Virus (HIV) would be valuable for the determination and evaluation of preventive treatments. Effective strategies are urgently needed to reduce mother to infant perinatal HIV transmission. Various ongoing or planned trials and studies concentrate on mode of infant feeding or antiretroviral therapy to the infant over the breastfeeding period. It is essential to determine the risk of perinatal HIV transmission. Further it is also essential to observe when the perinatal transmission takes place in infant. The main idea of modeling is to estimate the probabilities of positive polymerase chain reaction (PCR). The transmission of HIV from a HIV-positive mother to her child during pregnancy, labour, delivery or breastfeeding is called perinatal transmission. It results from fetal exposure to the maternal fluids or infected maternal secretions. The present article proposes the generalized statistical models that simultaneously estimate the risks of perinatal transmission together with the sensitivity of the screening tests for HIV infection. The article aims at brief overview of exponential and geometric distribution as lag time distributions. The methods are illustrated with the data from a randomized control study, conducted in South Africa.
\end{abstract}

Keywords: Human Immunodeficiency Virus (HIV); Transmission probabilities; Mother-to-infant HIV transmission; Perinatal HIV transmission; Maximum likelihood

\section{Introduction}

It is important to determine the risk of transmission of perinatal HIV transmission in medical study. It is also serious the time when perinatal HIV transmission occurs. From conception to delivery of infants there are three phases; antepartum, intrapartum and postpartum. Antepartum phase is period from conception to delivery, intrapartum phase is period during delivery and postpartum phase is period following the birth of infants. Perinatal HIV transmission can occur in one of the following phases; antepartum, intrapartum or postpartum. It is estimated that 90 percent HIV infected infants are infected through perinatal transmission (Raji Balasubramanian et.al.,2001). Throughout the world research is going on to find working methods to reduce perinatal HIV transmission. According to United Nations Program on HIV/ AIDS, an estimated 1800 HIV infected infants are born each day in the developing world. If HIV infected mother breastfed her infant, in the postpartum phase the risk of HIV transmission is possible at some extent. Estimation of the distribution of the time of perinatal transmission is difficult because tests of infection status can only be undertaken after birth. The present study is an extension of $\mathrm{N}$. Gupte et.al. (2007) work carried out as a randomized control study, conducted in Johannesburg, South Africa.

The present article is organized as follow: Section 2, gives the notations along with data description and also discusses the construction of likelihood for the underlying data. In section 3 , the different generalized models being proposed assuming various distributions for the lag times for estimation of the probability of perinatal HIV transmission. Section 4, is devoted to applications of proposed models to real life data and estimation of parameters. Further suitability of models is also discussed. Section 5 , gives the conclusions and comments about the modeling maternal infant HIV transmission.

\section{Generalized statistical modeling for perina- tal HIV transmission}

\subsection{Notations and Related Discussion}

In this section, notations required for the development of likelihood are introduced. If infection occurred in antepartum phase, let $\alpha$ denote the probability of HIV transmission to infant in antepartum phase. If transmission did not occur in antepartum phase then let $\beta$ denote the conditional probability of transmission during intrapartum phase. Therefore, in intrapartum phase the unconditional probability of transmission is $\beta(1-\alpha)$. After the birth, an uninfected infant is being breastfed at time t with $\lambda(t)$ be the hazard rate of infection through breastfeeding at time $t$. Infants were tested for HIV infection using viral assays such as polymerase chain reaction( PCR ).Under study, let $\mathrm{N}$ be the number of mother infant pairs, such that infants born to HIV infected mothers will be tested, using DNA PCR at the following time points $t_{1}$, $t_{2}, \ldots, t_{k}(k>1)$. Note that $t_{k}$ is $k^{\text {th }}$ the visit after breastfeeding discontinued to the infant. Let $\mathrm{O}_{\mathrm{i}}=\left(\mathrm{O}_{\mathrm{it}_{1}}, \mathrm{O}_{\mathrm{it}_{2}}, \ldots \mathrm{O}_{\mathrm{it}_{\mathrm{k}}}\right)$ denote observed results of DNA PCR assays at the scheduled visits $t_{1}$, $\mathrm{t}_{2}, \ldots \mathrm{t}_{\mathrm{k}}$ for $\mathrm{i}^{\text {th }}$ the infant, $\mathrm{i}=1,2, \ldots \mathrm{N}$. Note that, 
$\mathrm{O}_{\mathrm{ij}}=\left\{\begin{array}{l}1, \text { if DNA PCR is positive } \\ 0, \text { if DNA PCR is negative }\end{array}\right.$

$\mathrm{i}=1,2, \ldots, \mathrm{N}, \mathrm{j}=\mathrm{t}_{1}, \mathrm{t}_{2}, \ldots, \mathrm{t}_{\mathrm{k}}$.

If the $i^{t h}$ infant is breastfed then $\operatorname{td}_{\mathrm{i}}$ denote the time duration of breastfeeding. It is obvious to assume that if the $i^{\text {th }}$ infant is not breastfed then $t d_{i}=0$. The proportion of breastfed infants is considered to be $\omega$. The observed data are $\mathrm{O}=\left(\underline{\mathrm{O}}_{1}, \underline{\mathrm{O}}_{2}, \ldots, \underline{\mathrm{O}}_{\mathrm{N}}\right)$ and $\mathrm{D}=\left(\mathrm{td}_{1}, \mathrm{td}_{2}, \ldots \mathrm{td}_{\mathrm{N}}\right)$. Using DNA PCR results it is possible to identify all HIV-infected infants. The sensitivity of PCR may depend upon the timing of transmission and the time since infection. Our main interest is to study of sensitivity of PCR which is characterized by random variables $\mathrm{X}, \mathrm{Y}$ and $\mathrm{Z}$; which are defined as follow: If HIV transmission occurred in antepartum phase, let $\mathrm{X}$ be number of days after birth for positive PCR with $\mu_{\mathrm{x}}$ be the mean and $\mathrm{F}_{\mathrm{X}}($.$) be distribution function$ of X. Similarly, if transmission did not occur in antepartum period and it occurred in the intrapartum period then we define r.v. $\mathrm{Y}$ as number of days after birth for positive PCR with $\mu_{\mathrm{y}}$ be the mean and $\mathrm{F}_{\mathrm{y}}($.$) be distribution function of r.v.Y. Similarly, if transmis-$ sion occurred through breastfeeding, let $\mathrm{Z}$ be number of days after infection for a positive PCR with $\mu_{\mathrm{z}}$ be the mean and $\mathrm{F}_{\mathrm{z}}($.$) be dis-$ tribution function of r.v. Z. Random variables $\mathrm{X}, \mathrm{Y}$ and $\mathrm{Z}$ are considered as lag times for positive PCR assay.

\subsection{Likelihood Function}

In this section we construct a likelihood for the estimation of $\underline{\theta}=\left(\alpha, \beta, \lambda(\mathrm{t}), \mu_{\mathrm{x}}, \mu_{\mathrm{y}}, \mu_{\mathrm{z}}\right)$. Here $\underline{\theta}$ is the parameter of interest. If HIV-positive mothers breastfeed their infants, then one of the following cases may arise

Case 1: Infants who test positive at the first test, $t_{1}$

Case 2: Infants who test negative at $t_{j}$ and positive at $t_{j+1}$

$(\mathrm{j}=1,2, \ldots, \mathrm{k}-1)$

Case 3: Infants who test negative at $t_{k}$

The likelihood for each of the three cases is developed by $\mathrm{N}$ Gupte et al., (2007). The principle of construction of likelihood is discussed which is used to derive the likelihood under proposed models later. For Case 1, infants whose PCR is positive, the probabilities for breast-feeding (BF) and non-breast -feeding (NBF) are given as:

$$
\begin{aligned}
& p_{t_{1}}^{B F}(\underline{\theta})=\omega(1-\alpha)(1-\beta) \int_{0}^{\min \left\{t_{1}, t d_{i}\right\}} \lambda(x) \exp \left\{-\int_{0}^{x} \lambda(s) d s\right\} * \\
& F_{z}\left(t_{1}-x\right) d x+\omega\left[\alpha F_{x}\left(t_{1}\right)+(1-\alpha) \beta F_{y}\left(t_{1}\right)\right]
\end{aligned}
$$

For non- breast-feeding infants this probability is

$p_{t_{1}}^{N B F}(\underline{\theta})=(1-\omega)\left[\alpha F_{x}\left(t_{1}\right)+(1-\alpha) \beta F_{y}\left(t_{1}\right)\right]$

Similarly, for Case 2, infants whose HIV status by PCR is negative at time $t_{j}$, and positive at time $t_{j+1}$ the probabilities for breastfeeding and non- breast-feeding infants are given by

$$
\begin{aligned}
& p_{t_{j} t_{j+1}}^{B F}=\omega(1-\alpha)(1-\beta) \int_{0}^{\min \left\{t_{j}, t d_{i}\right\}} \lambda(x) \exp \left\{-\int_{0}^{x} \lambda(s) d s\right\} \\
& *\left[F_{z}\left(t_{j+1}-x\right)-F_{z}\left(t_{j}-x\right)\right] d x+ \\
& \int_{t_{j}}^{\min \left\{t_{j+1}, t d_{i}\right\}} \lambda(x) \exp \left\{-\int_{0}^{x} \lambda(s) d s\right\} F_{z}\left(t_{j+1}-x\right) d x+ \\
& \omega\left[\alpha\left(F_{x}\left(t_{j+1}\right)-F_{x}\left(t_{j}\right)\right)+(1-\alpha) \beta\left(F_{y}\left(t_{j+1}\right)-F_{y}\left(t_{j}\right)\right]\right.
\end{aligned}
$$

$$
\begin{aligned}
p_{t_{j} t_{j+1}}^{N B F}= & (1-\omega)\left[\alpha\left(F_{x}\left(t_{j+1}\right)-F_{x}\left(t_{j}\right)\right)+(1-\alpha) \beta\left(F_{y}\left(t_{j+1}\right)-\right.\right. \\
& \left.\left.F_{y}\left(t_{j}\right)\right)\right]
\end{aligned}
$$

respectively. Finally, for Case 3, infants whose PCR is negative at $t_{k}$, the probabilities for breastfeeding and non- breast-feeding infants are given by

$$
\begin{aligned}
p_{t_{k}}^{B F}(\underline{\theta})=\omega(1-\alpha) & (1-\beta) \int_{0}^{\min \left\{t_{k}, t d_{i}\right\}} \lambda(x) \exp \left\{-\int_{0}^{x} \lambda(s) d s\right\} \\
& *\left\{1-F_{z}\left(t_{k}-x\right)\right\} d x+1 \\
& -\int_{0}^{\min \left\{t_{k}, t d_{i}\right\}} \lambda(x) \exp \left\{-\int_{0}^{x} \lambda(s) d s\right\} \\
& * F_{z}\left(t_{j+1}-x\right) d x+\omega\left[\alpha\left(1-F_{x}\left(t_{k}\right)\right)\right. \\
& +(1-\alpha) \beta\left(1-F_{y}\left(t_{k}\right)\right]
\end{aligned}
$$

$$
\begin{aligned}
\mathrm{p}_{\mathrm{t}_{\mathrm{k},},}^{\mathrm{NBF}}(\underline{\theta})= & (1-\omega)\left[\alpha\left(1-F_{x}\left(t_{k}\right)\right)+(1-\alpha) \beta\left(1-F_{y}\left(t_{k}\right)+\right.\right. \\
& (1-\alpha)(1-\beta)]
\end{aligned}
$$

From the total infants under study, for Case 1 , let $\mathrm{n}_{\mathrm{t}_{1}}^{\mathrm{BF}}$ infants were breastfed and $\mathrm{n}_{\mathrm{t}_{1}}^{\mathrm{NBF}}$ were not breastfed infants who test positive at $t_{1}$. For Case 2, let $n_{t_{j} t_{j+1}}^{B F}$ be breastfed infants and $n_{t_{j} t_{j+1}}^{N B F}$ nonbreast-fed infants who test negative at $t_{j}$ and positive at $t_{j+1}$. For Case 3, let $\mathrm{n}_{\mathrm{k}}^{\mathrm{BF}}$ be breastfed infants and $\mathrm{n}_{\mathrm{t}_{\mathrm{k}}}^{\mathrm{NBF}}$ be non -breastfed infants who test negative at time $t_{k}$. Considering all the cases, theexpression for likelihood function for estimating $\underline{\theta}$ is,

$$
\begin{aligned}
L(\underline{\theta} \mid O, D)=\left[p_{t_{1}}^{B F}(\underline{\theta})\right]^{n_{t_{1}}^{B F}} *\left[p_{t_{1}}^{N B F}(\underline{\theta})\right]^{n_{t_{1}}^{N B F}} & \\
& * \prod_{j=1}^{k-1}\left[p_{t_{j}, t_{j+1}}^{B F}(\underline{\theta})\right]^{n_{\mathrm{t}_{j}}^{B F} t_{j+1}} * \prod_{j=1}^{k-1}\left[p_{t_{t_{j}}, t_{j+1}}^{N B F}(\underline{\theta})\right]^{n_{\mathrm{t}_{j}}^{N B F} t_{j+1}} \\
& *\left[p_{t_{k}}^{B F}(\underline{\theta})\right]^{n_{t_{k}}^{B F}} *\left[p_{t_{k}}^{N B F}(\underline{\theta})\right]^{n_{k}^{N B F}}
\end{aligned}
$$

\section{Proposed Models}

In the further discussion we proposed various models for HIV transmission based on various lag time distributions. As pointed out earlier these are based on the various distributional assumptions for the lag time random variables $\mathrm{X}, \mathrm{Y}$ and $\mathrm{Z}$. Initially we will discuss the form of likelihood under lag time distributions as an exponential distribution and results are revisited as those of $(\mathrm{N}$. Gupte el al.,2007). However strictly speaking the variables X, Y and $\mathrm{Z}$ can be regarded as discrete random variables hence it is most appropriate to assume some discrete distributions for random variables $\mathrm{X}, \mathrm{Y}$ and $\mathrm{Z}$. With this thought in mind it is decided to discuss the analysis by assuming $\mathrm{X}, \mathrm{Y}$ and $\mathrm{Z}$ to be geometric random variables.

\subsection{Exponential Lag Time Distribution (Model - I) and Geometric Lag Time Distribution (Model - II)}

In the case of exponential distribution transmission rates were estimated on the basis of the following assumptions : (i) X, Y and $\mathrm{Z}$ have an exponential distribution with means $\boldsymbol{\mu}_{\boldsymbol{X}}, \boldsymbol{\mu}_{\boldsymbol{Y}}$ and $\boldsymbol{\mu}_{\boldsymbol{Z}}$ respectively (ii) hazard rate to be constant $\lambda$ per month (iii) $\min \left\{\mathrm{t}_{1}\right.$, $\left.\mathrm{td}_{\mathrm{i}}\right\}=\mathrm{t}_{1}, \min \left\{\mathrm{t}_{\mathrm{j}}, \mathrm{td}_{\mathrm{i}}\right\}=\mathrm{t}_{\mathrm{j}}$, and $\min \left\{\mathrm{t}_{\mathrm{k}}, \mathrm{td}_{\mathrm{i}}\right\}=\mathrm{t}_{\mathrm{k}}$. The likelihood for this model will be maximized numerically using Wolfram Mathematica 10.0. Similarly in case of geometric distribution rates were estimated on the basis of the following assumptions : (i) $\mathrm{X}$, $\mathrm{Y}$ and $\mathrm{Z}$ have geometric distribution with parameters $\mathrm{p}_{\mathrm{x}}, \mathrm{p}_{\mathrm{y}}$ and $\mathrm{p}_{\mathrm{z}}$ respectively (ii) hazard rate to be constant $\lambda$ per month (iii) $\min \left\{\mathrm{t}_{1}, \mathrm{td}_{\mathrm{i}}\right\}=\mathrm{t}_{1}, \min \left\{\mathrm{t}_{\mathrm{j}}, \mathrm{td}_{\mathrm{i}}\right\}=\mathrm{t}_{\mathrm{j}}$, and $\min \left\{\mathrm{t}_{\mathrm{k}}, \mathrm{td}_{\mathrm{i}}\right\}=\mathrm{t}_{\mathrm{k}}$. The likelihood for this model also maximized numerically using Wolfram Mathematica 10.0 to obtain the estimates of the parameters. 


\section{Applications of Proposed Models to Real Life Data}

In South African Study, infants admitted to Chris Hani Baragwanath, coronation Neonatal Unit. As a part of treatment, Infants of all consenting mothers were randomized to receive either six weeks of AZT or one dose of NVP. Infants were given the medication within 24 hours of post-delivery. Under study, there were one thousand and forty mother infant pairs enrolled. Out of which 461 mothers breastfed their infants and 512 mothers selected an alternative feeding pattern mechanism. At the post-partum visits, HIV infection status for infants was determined using DNA PCR. The postpartum visits are performed at birth $3^{\text {rd }}$ day, $10^{\text {th }}$ day, $42^{\text {nd }}$ day, $90^{\text {th }}$ day and $180^{\text {th }}$ day. The likelihood func$\operatorname{tionL}(\underline{\boldsymbol{\theta}} \mid \mathbf{O}, \mathbf{D})$ was maximized numerically using Wolfram Mathematica 10.0 to obtain the estimate $\underline{\widehat{\boldsymbol{\theta}}}$

\subsection{Estimation of $\underline{\theta}$}

Transmission rates are estimated using these models. Further using the fitted models, we have also estimated the expected proportion of HIV - positive infants at each post-partum visit.

Table 1: Lag Time distribution of X, Y, Z: Exponential distribution: Model-I

\begin{tabular}{|c|c|c|}
\hline$\widehat{\alpha}=0.1085$ & $\widehat{\beta}=0.05481$ & $\widehat{\lambda}=0.00000021$ \\
\hline$\widehat{\mu_{x}}=20.64$ & $\widehat{\mu_{y}}=0.1674$ & $\widehat{\mu_{z}}=3.04564$ \\
\hline$\widehat{t_{t_{1}}}=0.06354$ & $\widehat{t_{t_{2}}}=0.09053$ & $\widehat{p_{t_{3}}}=0.1432$ \\
\hline$\widehat{p_{t_{4}}}=0.15601$ & $\widehat{p_{t_{5}}}=0.1573$ & \\
\hline
\end{tabular}

Table 2: Lag Time distribution of X, Y, Z: Geometric distribution: Model - II

\begin{tabular}{|c|c|c|}
\hline$\widehat{\alpha}=0.8408$ & $\hat{\beta}=0.7026$ & $\widehat{\lambda}=0.0923$ \\
\hline$\widehat{q_{x}}=0.999$ & $\widehat{q_{y}}=0.8146$ & $\widehat{q_{z}}=0.9156$ \\
\hline$\widehat{p_{t_{1}}}=0.0527$ & $\widehat{p_{t_{2}}}=0.1048$ & $\widehat{p_{t_{3}}}=0.1414$ \\
\hline$\widehat{p_{t_{4}}}=0.1549$ & $\widehat{p_{t_{5}}}=0.1752$ & \\
\hline
\end{tabular}

With $q_{x}=1-p_{x}, q_{y}=1-p_{y}$ and $q_{z}=1-p_{z}$

Table 3: AIC, BIC and AIC corrected for Model I and II

\begin{tabular}{|c|c|c|c|}
\hline Model & AIC & BIC & AIC corrected \\
\hline I & 2347.18 & 2376.44 & 2347.26 \\
\hline II & 2368.66 & 2397.92 & 2368.74 \\
\hline
\end{tabular}

\section{Conclusion}

On the basis of AIC and BIC results the model with lag time distribution as exponential (Model -I) performs better as compared to geometric (Model- II) model.(refer Table 3). The Model with minimum absolute difference between observed probability and estimated probability of positive PCR, that Model is suggested for real life data. The maximum likelihood estimators (m.l.e) so obtained are consistent solution of the likelihood equation. It is explored that the estimation of the parameter is under assumptions the likelihood admits consistent and unique solution. Consistent in a sense that the solution converges in probability to the true value $\theta$. As per the results of Cramer - Huzurbazar theory, it is verified that the assumptions regarding maximum likelihood estimators (m.l.e) obtained are consistent solution of the likelihood equation using Wolfram Mathematica 10.0 (details not shown) .This models underestimates the proportions of perinatal HIV transmission at birth but over estimates the transmission probabilities at subsequent visits. We have proposed models considering lag time distributions to be exponential and geometric. Further as the results are encouraging authors also worked with shifted-geometric distribution as lag time distribution.

\section{References}

[1] A review of available evidence, (2004) WHO Library Cataloguing in- Publication Data HIV transmission through breastfeeding, A review of available evidence
[2] Paolo, G.M, Taha, E.T, et al, (1999), HIV transmission through breastfeeding, A study in Malawi. Journal of American Medical Association 282-2, 744-749

[3] N. Gupte, R. Brookmeyer, et al, (2007), Modeling Maternal - Infant HIV Transmission in the presence of breastfeeding with an imperfect test. Biometrics 63, 1189-1197

[4] Newton L.k., Hoover D.R, et al, (2008), Extended Antiretroviral Prophylaxis to reduce breast-milk HIV-1 transmission, The New England Journal of Medicine 359, 119-129

[5] Raji Balasubramanian and Lagakos S.W., (2001), Estimation of timing of perinatal transmission of HIV. Biometrics $57,1048-1058$

[6] Raji Balasubramanian and Lagakos S.W. (2003), Estimation of a failure time distribution based on imperfect diagnostic tests. Biometrika 9, 171-182

[7] Subhash Shende and Mohan Kale, et al (2017), Modeling Maternal -Infant HIV Transmission with Lag Time Distributions Exponential, Geometric and Shifted Geometric. Neural, Parallel, and Scientific Computations 25, 509-522 